\title{
Dynamic assembly of silent chromatin during thymocyte maturation
}

\author{
Ruey-Chyi Su ${ }^{1}$, Karen E Brown ${ }^{2}$, Sanam Saaber ${ }^{1}$, Amanda G Fisher ${ }^{2}$, Matthias Merkenschlager ${ }^{2}$ \& Stephen T Smale ${ }^{1}$
}

Considerable knowledge has been gained from temporal analyses of molecular events culminating in gene activation 1 , but technical hurdles have hindered comparable studies of gene silencing. Here we describe the temporal assembly of silent chromatin at the mouse terminal transferase gene (Dntt), which is silenced and repositioned to pericentromeric

heterochromatin during thymocyte maturation². Silencing was nucleated at the Dntt promoter by the ordered deacetylation of histone H3 at Lys9 (H3-Lys9), loss of methylation at H3-Lys4 and acquisition of methylation at H3-Lys9, followed by bidirectional spreading of each event. Deacetylation at H3-Lys9 coincided with pericentromeric repositioning, and neither of these early events required de novo protein synthesis. CpG methylation increased primarily in mature $\mathrm{T}$ cells that had left the thymus. A transformed thymocyte line supported reversible inactivation of Dntt without repositioning. In these cells, histone modification changes were nucleated at the promoter but did not spread. These results provide a foundation for elucidating the mechanisms of silent chromatin assembly during development.

Although our knowledge of the properties of silent DNA and of the assembly of silent chromatin has increased ${ }^{3-11}$, direct temporal analyses of silent chromatin assembly have been limited largely to mammalian X-chromosome inactivation ${ }^{12-16}$. The insights provided by

Figure 1 Reversible versus irreversible silencing of Dntt transcription during thymocyte differentiation. (a) The thymus of a young mouse contains large quantities of double-positive thymocytes. Efficient maturation ex vivo results in irreversible silencing of Dntt, Rag1 and Rag2 and repositioning of their alleles to pericentromeric foci. Differentiation of the VL3-3M2 thymocyte line is accompanied by reversible inactivation of Dntt, Rag1 and Rag2 transcription, which can be restored by reculturing in the absence of the stimulus. (b) The transition from reversible to irreversible silencing of Dntt during maturation of primary thymocytes and (c) the absence of irreversible silencing in VL3-3M2 cells are shown. Thymocytes (b) or cells (c) stimulated with PMA plus ionomycin for 4-12 $\mathrm{h}$ were washed and recultured in the absence of stimulus for $4-12 \mathrm{~h}$. Transcripts were analyzed by RT-PCR. genetic models and the $\mathrm{X}$ inactivation model have been invaluable, but little is known about the silencing of individual developmentally regulated genes. One reason for this paucity of knowledge is that few developmental pathways are amenable to direct temporal analysis. Large quantities of immature primary cells are needed for a temporal analysis using common molecular assays, and it must be possible to induce synchronous and rapid gene silencing in a high percentage of cells in the population.

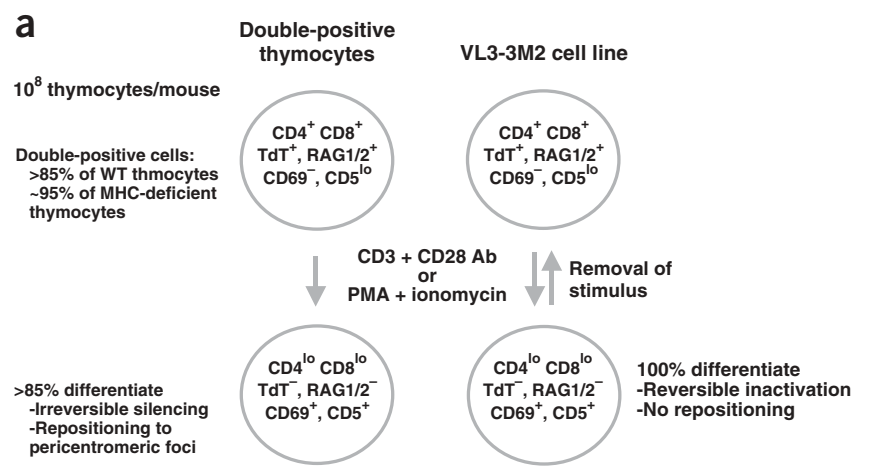

b

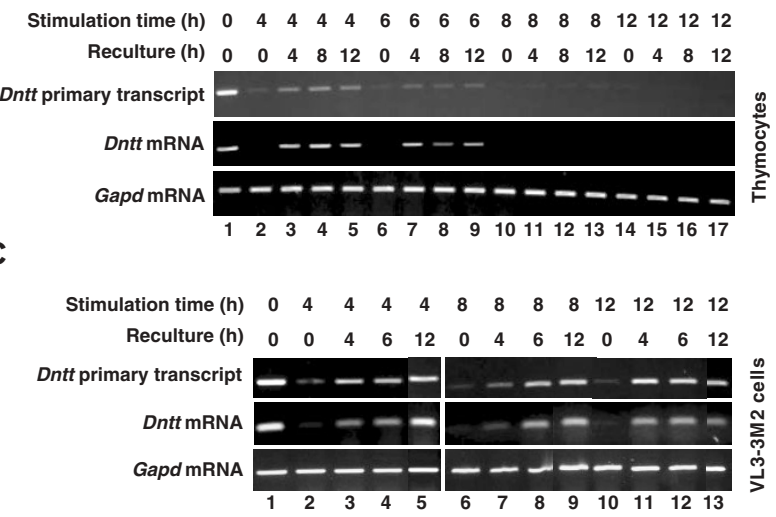

${ }^{1}$ Howard Hughes Medical Institute and Department of Microbiology, Immunology, and Molecular Genetics, University of California, Los Angeles, California 900951662, USA. ${ }^{2}$ Lymphocyte Development Group, MRC Clinical Sciences Centre, Imperial College School of Medicine, Hammersmith Hospital, London W120NN, UK. Correspondence should be addressed to S.T.S. (smale@mednet.ucla.edu). 
Double-positive mouse thymocytes serve as a good model for studying the temporal events associated with silencing. These abundant immature cells actively express Rag1 and Rag2, which encode the $\mathrm{V}(\mathrm{D}) \mathrm{J}$ recombinase, and $\mathrm{Dntt}$, whose product adds $\mathrm{N}$ regions at recombination junctions ${ }^{17}$. After recombination of the gene Tcra, the T-cell receptor (TCR) interacts with peptide-associated major histocompatibility complex (MHC) molecules, triggering thymocyte maturation. The early events associated with maturation, including silencing of Dntt, can be recapitulated ex vivo by stimulation with TCR complex antibodies or phorbol myristate acetate (PMA) plus ionomycin (Fig. 1a) ${ }^{2,18-21}$. In MHC-deficient mice, $~ 95 \%$ of thymocytes are at the double-positive stage and ex vivo stimulation results in efficient maturation (Supplementary Fig. 1 online). Most thymocytes do not proliferate ex vivo, which limits our ability to monitor the heritability of silencing. We therefore focus on the property of 'irreversible' silencing, which we define as the absence of spontaneous transcriptional reactivation after removal of the stimulus used to trigger maturation.

RT-PCR analysis showed that inactivation of Dntt remained largely reversible $6 \mathrm{~h}$ after stimulation (Fig. 1b), but reversibility was mostly lost by the 8-h time point. The transformed VL3-3M2 thymocyte line supports this same developmental step after stimulation (Supplementary Fig. 1 online), but Dntt inactivation remained reversible $12 \mathrm{~h}$ (Fig. 1c) or $18 \mathrm{~h}$ (data not shown) after stimulation $^{2,19,21}$

CpG methylation increased only slightly at the Dntt locus after thymocyte stimulation (Supplementary Fig. 2 online). We observed large increases in CpG methylation only in mature $\mathrm{T}$ cells that had migrated to the spleen and had been stimulated to proliferate. Restriction enzyme accessibility at the Dntt promoter gradually decreased between 0.5 and $8 \mathrm{~h}$ after stimulation in nuclei from primary thymocytes and VL3-3M2 cells (Supplementary Fig. 3 online). Reduced accessibility may reflect the loss of a remodeled nucleosomal state at the promoter.

\section{a}

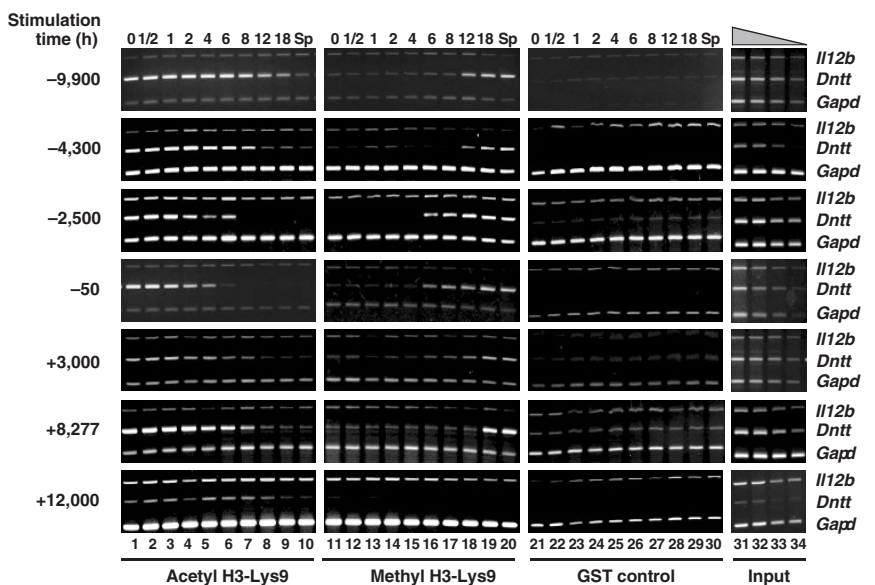

Using chromatin immunoprecipitation (ChIP), we observed efficient deacetylation at H3-Lys9 at the Dntt promoter in primary and transformed thymocytes (Fig. 2a,b). Deacetylation was followed by a marked increase in methylation at H3-Lys9, as shown by using an antibody raised against dimethylated Lys9 (which may not distinguish dimethylated from trimethylated Lys9 or methylated Lys9 from methylated Lys27). Because methylation at H3-Lys9 at the promoter increased in both primary and transformed cells, this event is probably not sufficient for irreversibility. In VL3-3M2 cells, we observed a substantial increase in acetylation and decrease in methylation at H3-Lys9 after removing the PMA plus ionomycin stimulus (Fig. 3a), which confirms that deacetylation and methylation remained reversible.

The above results did not show a difference between reversible inactivation and irreversible silencing, but we noted a marked difference when we analyzed other regions of the Dntt locus. In primary thymocytes, deacetylation and methylation of H3-Lys9 extended several kilobases upstream and downstream of the promoter (Fig. 2a). The time courses suggest that these events spread bidirectionally after nucleation in close proximity to the Dntt promoter. Spreading was not detected in VL3-3M2 cells (Fig. 2b), which may explain the absence of irreversibility.

The differences in acetylation and methylation were much more pronounced at Dntt than at the constitutively active and silent genes Gapd and Il12b (Fig. 2). Although we observed modest biases towards acetylation or methylation in some experiments, the biases were much less pronounced than those at Dntt. We speculate that histone modifications may be polarized more strongly at actively regulated genes than at constitutively active or silent genes.

Quantification by real-time PCR showed that deacetylation at H3-Lys9 at the Dntt promoter occurred rapidly, with the most substantial decrease occurring 2-6 h after stimulation (Fig. 3b). We also monitored methylation at H3-Lys4 in these experiments and observed an efficient loss of methylation 4-12 h after stimulation

\section{b}

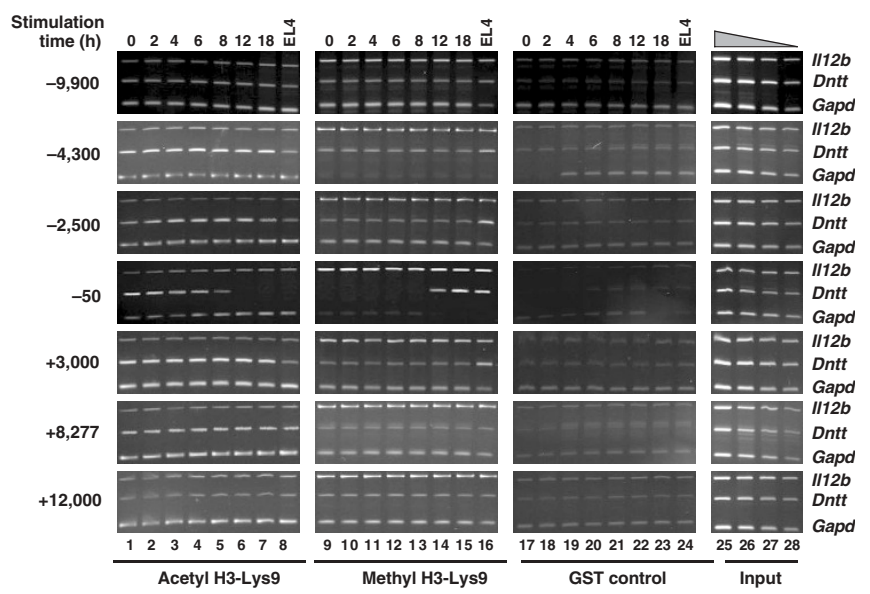

Figure 2 Promoter nucleation and bidirectional spreading of deacetylation and methylation at H3-Lys9 in thymocytes but not in VL3-3M2 cells. (a) MHCdeficient thymocytes were stimulated with PMA plus ionomycin for 0-18 h. Deacetylation (lanes 1-9) and methylation (lanes 11-19) at H3-Lys9 were monitored using a ChIP assay. PCR primers corresponded to seven regions of the Dntt locus extending $9.9 \mathrm{~kb}$ upstream and $12 \mathrm{~kb}$ downstream of the transcription start site. Chromatin samples from resting splenocytes, in which Dntt was silent, were also analyzed (Sp, lanes 10, 20 and 30). The silent gene $1 / 12 b$ and active gene Gapd were analyzed as controls. The relative strengths of the Gapd and $/ / 12 b$ signals varied from experiment to experiment and when coupled to different primer pairs from the Dntt locus but were generally consistent within a given experiment. Control antibodies were directed against glutathione-S-transferase (GST, lanes 21-30). (b) VL3-3M2 cells were stimulated with PMA plus ionomycin for 0-18 h. Deacetylation and methylation at H3-Lys9 were monitored as described above. Chromatin samples from EL4 cells, in which Dntt was silent, were also analyzed (lanes 8, 16 and 24). 
(Fig. 3b). Methylation at H3-Lys9 was most efficient 6-18 h after stimulation, consistent with evidence that methylation at H3-Lys9 is inhibited by methylation at H3-Lys4 (ref. 22). Temporal spreading of deacetylation throughout the Dntt locus was readily apparent (Fig. 3c) and occurred at a rate of $\sim 2 \mathrm{~kb} \mathrm{~h}^{-1}$. Temporal spreading of the loss of methylation at H3-Lys4 was not apparent (Fig. 3e). The H3-Lys4 methyl group either was lost too rapidly for detection of spreading or was simultaneously lost throughout the deacetylated locus. Notably, the loss of methylation at H3-Lys4 throughout a 22$\mathrm{kb}$ region in nonproliferative cells indicates that an efficient replication-independent mechanism must exist for the removal of this modification. Bidirectional spreading of methylation at H3-Lys9 was readily apparent (Fig. $\mathbf{3 g}$ ), with the nucleation site in close proximity to the promoter.

We obtained additional insight by determining the relative DNA concentrations in each sample by normalizing the amplification efficiencies of the different primer pairs (Fig. 3d,f,h). In unstimulated cells, the densities of acetylation at H3-Lys9 and methylation at H3-Lys4 were highest at the Dntt promoter. The density of methylation at H3-Lys9 after stimulation was also highest at the promoter. These results suggest that the efficiency with which the modification machinery can catalyze spreading has intrinsic limits.

RT-PCR analysis of Dntt transcripts in cells stimulated in the presence of cycloheximide (CHX) showed that downregulation does not require protein synthesis (Fig. 4a). Deacetylation was also independent of protein synthesis, but protein synthesis was essential for the loss of methylation at H3Lys4 and acquisition of methylation at H3-

Figure 3 Quantitative analysis of deacetylation and methylation at H3-Lys9 and of the loss of methylation at H3-Lys4. (a) Deacetylation and methylation at H3-Lys9 remained reversible at the Dntt promoter in VL3-3M2 cells. VL3-3M2 cells stimulated with PMA plus ionomycin for 12 or $16 \mathrm{~h}$ were washed and then cultured in the absence of stimulus for 4 or $12 \mathrm{~h}$. Histone $\mathrm{H} 3$ modifications were monitored by ChIP. (b) Temporal changes in histone modification state at the Dntt promoter were monitored by real-time ChIP, using antibodies against acetylated H3-Lys9, dimethylated H3-Lys9 and dimethylated H3-Lys4. Time points between 0 and $18 \mathrm{~h}$ were analyzed. The 24- $\mathrm{h}$ values correspond to the relative values observed in resting splenocytes, which corresponds to lymphocytes in which Dntt had been silent for a long period of time. The $100 \%$ value corresponds to the 0-h time point for acetylated H3-Lys9 and methylated H3-Lys4 and to the splenocyte value for methylated H3-Lys9. (c,e,g) Changes in histone modification at various regions of the Dntt locus were determined by real-time ChIP, using thymocytes stimulated for $0-18 \mathrm{~h}$. The 24 -h values correspond to the relative values observed in resting splenocytes. The $100 \%$ value corresponds to the 0-h time point for acetylated H3-Lys9 and methylated H3-Lys4 and to the splenocyte value for methylated H3-Lys9. (d,f,h) Relative DNA concentrations in the ChIP samples were determined by normalizing for the amplification efficiencies of the various primer pairs.
Lys9 (Fig. 4b,c). The experiments in Figure 4c used antibodies that recognize trimethylated $\mathrm{H} 3$-Lys9/Lys 27 and trimethylated H3-Lys4, indicating that a portion of the signal detected in the previous experiments was due to trimethylation. Dntt inactivation remained reversible in thymocytes stimulated for $12 \mathrm{~h}$ in the presence of $\mathrm{CHX}$ (data not shown), indicating that protein synthesis is required for irreversible silencing.

Fluorescence in situ hybridization (FISH) experiments uncovered the temporal relationship between pericentromeric repositioning and the histone modification changes (Fig. 4d). Association with the $\gamma$-satellite foci was preceded by a prevalent state in which the Dntt alleles were in close proximity but not directly coincident with the $\gamma$ satellite signal (referred to as 'constrained'). The frequent occurrence of constrained alleles at short times after stimulation suggests that they represent a true transitional state.

After stimulation with TCR antibodies, repositioning occurred $0.5 \mathrm{~h}$ after stimulation and reached a plateau by $6 \mathrm{~h}$ (Fig. $4 \mathrm{~d})$. After stimulation with PMA plus ionomycin, repositioning was more efficient. The percentage of cells in which both alleles were associated or constrained continued to increase between 6 and $12 \mathrm{~h}$, but substantial repositioning occurred before methylation at H3-Lys9 or the loss a

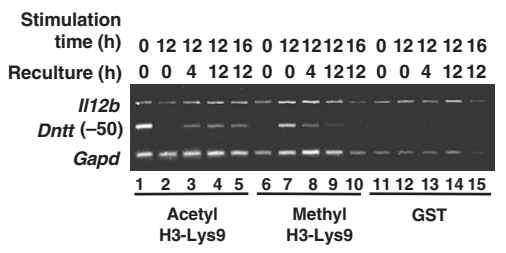

C

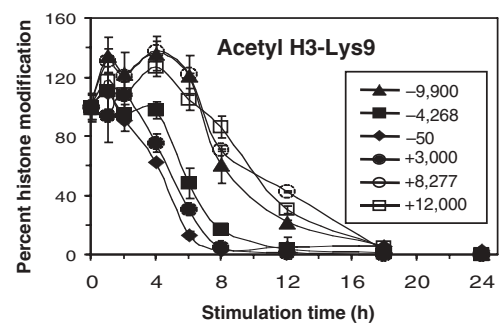

e

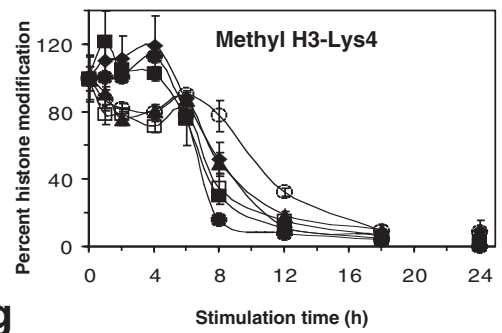

g

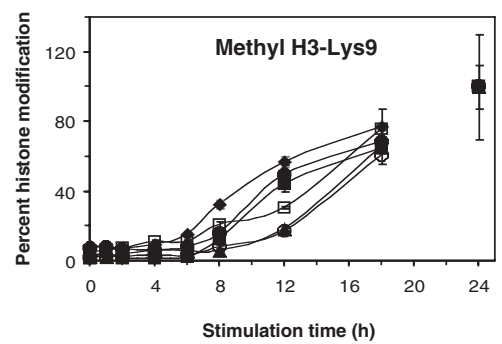

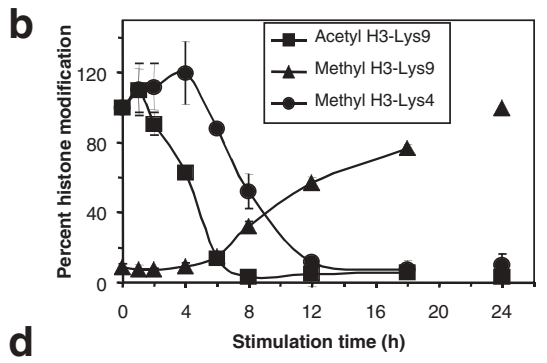

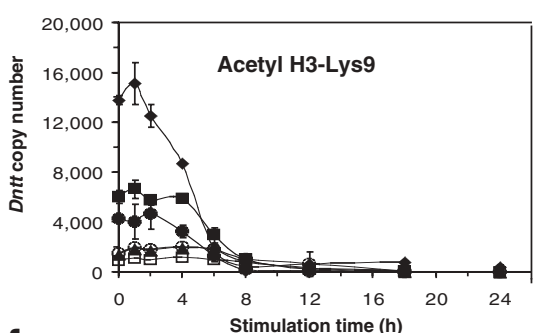

f
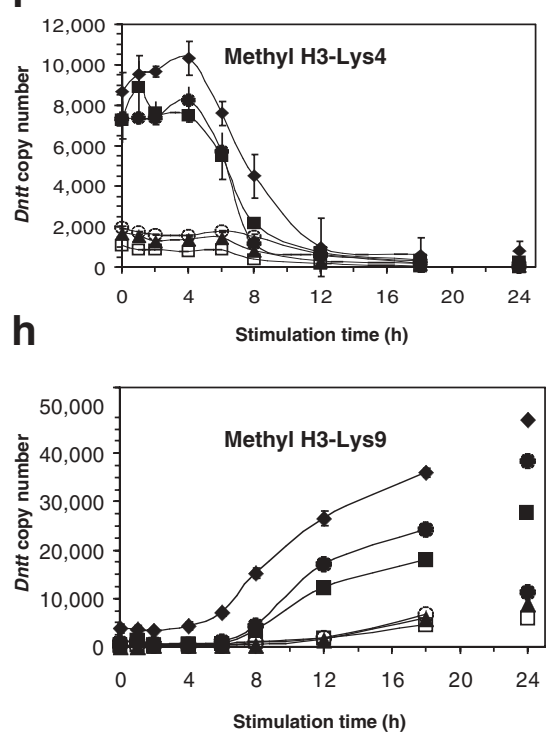
of methylation at H3-Lys4. At the 3-h time point, CHX had no effect on repositioning, indicating that protein synthesis is not required. At $12 \mathrm{~h}$, we observed a substantial decrease in repositioned alleles in the presence of $\mathrm{CHX}$, suggesting that protein synthesis is required for maintaining association with pericentromeric foci.

For many years, lymphocytes have served as good models for studying developmental regulation because cells maintained at specific developmental stages are relatively easy to isolate. Double-positive thymocytes are useful for studying gene silencing because the genes encoding the $\mathrm{V}(\mathrm{D}) \mathrm{J}$ recombination machinery are efficiently inactivated in vivo and ex vivo. The events uncovered here are similar to those involved in the assembly of constitutive heterochromatin in Schizosaccharomyces pombe and of facultative heterochromatin during $\mathrm{X}$ inactivation ${ }^{7,13}$. One clear difference, however, is that deacetylation and methylation at H3-Lys9 are nucleated at the Dntt promoter rather than within repetitive DNA elements. The requirement for the RNA-interference machinery during silent chromatin assembly in $S$. pombe has been linked to transcripts derived from repetitive elements ${ }^{7}$. Therefore, the RNAi pathway may not be required for Dntt silencing. Because Ikaros binding sites within the Dntt promoter are required for transcriptional downregulation in

\section{a}

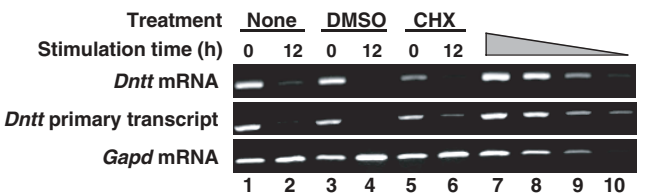

b

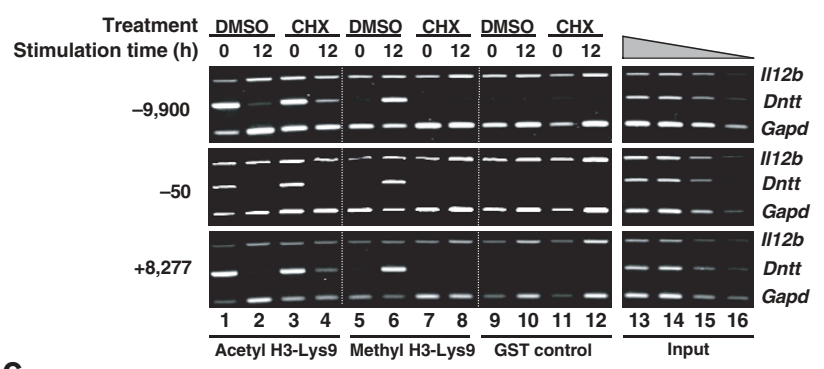

C

Treatment DMSO CHX DMSO CHX DMSO CHX

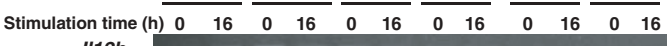
II12b

Dntt (-50) Gapd $-------1$ $\begin{array}{llllllllllll}1 & 2 & 3 & 4 & 5 & 6 & 7 & 8 & 9 & 10 & 11 & 12\end{array}$

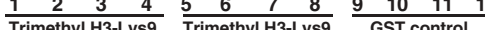
(13) $\begin{array}{llll}13 & 14 & 15 & 16\end{array}$

d Red: $\gamma$-satellites
Green: Dntt locus
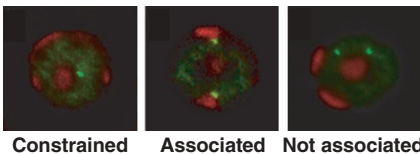

Constrained Associated Not associated

\section{Stimulation} time (h)

TCR Ab

$$
\text { 苐 }
$$

Both alleles assoc. or constr.

One allele assoc. or constr.

Neither allele assoc. or constr.

PMA + lono.

屯ัँ

Both alleles assoc. or const.

One allele assoc. or constr.

Neither allele assoc. or constr.

\begin{tabular}{cccccc}
0 & $1 / 2$ & 1 & 3 & 6 & 20 \\
\hline 0 & 0 & 0 & 13 & 31 & 33 \\
0 & 6 & 22 & 43 & 35 & 45 \\
100 & 94 & 78 & 44 & 34 & 22
\end{tabular}

$\begin{array}{llllll}0 & 3 & 3(\mathrm{CHX}) & 6 & 12 & 12(\mathrm{CHX})\end{array}$

$\begin{array}{llllll}0 & 18 & 20 & 23 & 52 & 11 \\ 0 & 47 & 46 & 60 & 33 & 26\end{array}$

$\begin{array}{llllll}0 & 47 & 46 & 60 & 33 & 26\end{array}$

VL3-3M2 cells ${ }^{21}$, and because Ikaros associates with the NuRD histone deacetylase complex ${ }^{23}$, Ikaros and NuRD may help nucleate deacetylation at H3-Lys9.

Our results show that the nucleation of deacetylation and methylation at H3-Lys9 can be separated from their spreading. The reason for the spreading deficiency in VL3-3M2 cells is not known, but this deficiency may provide an opportunity to study the spreading mechanism. Our results also showed that deacetylation is readily separated from the loss of methylation at H3-Lys4 and from methylation at H3-Lys9. These observations show that the removal of the acetyl group at H3-Lys9 is not sufficient for the subsequent events. Among the most notable observations were the early repositioning to pericentromeric foci and the efficient loss of methylation at H3-Lys4 throughout a $22-\mathrm{kb}$ region in the absence of proliferation. This latter finding suggests that histone exchange can occur in an efficient manner throughout a large genomic region, or that efficient demethylation or histone $\mathrm{H} 3$ tail clipping can occur during thymopoiesis. Most importantly, the rapid and synchronous assembly of silent chromatin that we observed suggests that double-positive thymocytes may serve as a valuable model for further elucidation of the assembly pathway in vivo and, ultimately, in vitro.

\section{METHODS}

Cells. We isolated thymocytes from MHC-deficient mice (4-8 weeks old $)^{24}$ and cultured them $\left(3-4 \times 10^{7}\right.$ cells per $10-\mathrm{cm}$ dish $)$ in RPMI 1640 medium (Mediatech) containing 10\% fetal calf serum, 2-mercaptoethanol, L-glutamate and antibiotics. We maintained the VL3-3M2 double-positive thymocyte line and EL4 mature thymocyte line in the same medium. We stimulated VL3-3M2 cells using PMA (50 $\mathrm{ng} \mathrm{ml}^{-1}$ ) plus ionomycin $\left(700 \mathrm{ng} \mathrm{ml}^{-1}\right)$. We stimulated primary thymocytes with PMA $\left(7.5 \mathrm{ng} \mathrm{ml}^{-1}\right)$ plus ionomycin $\left(180 \mathrm{ng} \mathrm{ml}^{-1}\right)$ or by plating on culture dishes coated with CD3 and CD28 antibodies (clones 1452C11 and 37.51; Pharmingen). We prepared splenocytes from C57BL/6 mice (4-8 weeks old). Mouse protocols were approved by the UCLA Chancellor's Animal Research Committee.

RT-PCR and ChIP assays. We prepared total RNA using TRI-reagent (Molecular Research Center). We resuspended the RNA pellet in water $(200 \mu \mathrm{l})$ and digested it with RNase-free DNase I (Pharmingen) for $30 \mathrm{~min}$ at $37^{\circ} \mathrm{C}$. We removed DNase I with acidic phenol-chloroform extraction (Sigma), followed by precipitation. We then reverse-transcribed RNA into cDNA with sequencespecific primers and amplified the resulting cDNA by PCR with primer sets specific to Dntt primary transcripts, Dntt mRNA or Gapd mRNA.

Figure 4 Effect of $\mathrm{CHX}$ on Dntt silencing, histone modification changes and pericentromeric repositioning. (a) MHC-deficient thymocytes were stimulated with PMA plus ionomycin for 0 or $12 \mathrm{~h}$ after a 3-h pretreatment and in the continual presence of $\mathrm{CHX}$ dissolved in dimethylsulfoxide (lanes 5 and 6), of dimethylsulfoxide alone (DMSO; lanes 3 and 4) or with no additive (lanes 1 and 2). Transcripts were monitored as in Figure 1. (b) MHC-deficient thymocytes were stimulated with PMA plus ionomycin and $\mathrm{CHX}$ as in $\mathbf{a}$. Deacetylation and methylation at H3-Lys9 were monitored using the ChIP assay. (c) MHC-deficient thymocytes were stimulated with PMA plus ionomycin and $\mathrm{CHX}$ as in a. Trimethylation at H3-Lys9/Lys27 and the loss of trimethylation at H3-Lys4 were monitored using the ChIP assay and antibodies that are specific for these modifications. (d) MHC-deficient thymocytes were stimulated with TCR antibodies for $0-20 \mathrm{~h}$ or with PMA plus ionomycin for 0-12 h. CHX sensitivity was monitored at 3 and $12 \mathrm{~h}$. The subnuclear locations of the endogenous Dntt alleles were determined by FISH. The percentages of cells in which both alleles, one allele or neither allele were either associated with pericentromeric foci or in a constrained state are indicated. Alleles were defined as constrained if they were within or at the edge of the dark area surrounding the $\gamma$-satellite foci (which was less accessible to background staining) and if the long side of the elongated locus was parallel with the face of the $\gamma$-satellite signal. The components of this dark area and the significance of this constrained state are not known. 
We carried out semiquantitative and quantitative ChIP assays using modified versions of the manufacturer's protocol (Upstate Biotechnology). Detailed protocols are given in Supplementary Note online. The antibodies to acetylated H3-Lys9, dimethylated H3-Lys9 and dimethylated H3-Lys4 were from Upstate Biotechnology (catalog numbers 06-942, 07-212/05-685 and 07-030, respectively). The H3-Lys9 antibody may crossreact with trimethylated $\mathrm{H} 3-$ Lys9 and with dimethylated and trimethylated H3-Lys27, and the H3-Lys4 antibody may crossreact with trimethylated H3-Lys4. Antibodies specific for trimethylated H3-Lys9/Lys27 and trimethylated H3-Lys4 were from Abcam (ab8898 and ab8580, respectively).

Confocal microscopy. We carried out FISH experiments with $\gamma$-satellite and Dntt probes and stimulated thymocytes with TCR antibodies as previously described $^{2}$. We stimulated thymocytes with PMA plus ionomycin using the same conditions as in the ChIP experiments.

Note: Supplementary information is available on the Nature Genetics website.

\section{ACKNOWLEDGMENTS}

We thank S. Amoyles, D. Russell and V. Agarwal for assistance. This work was supported by a grant from the National Institutes of Health. S.T.S. is an Investigator with the Howard Hughes Medical Institute.

\section{COMPETING INTERESTS STATEMENT}

The authors declare that they have no competing financial interests.

Received 5 January; accepted 19 March 2004

Published online at http://www.nature.com/naturegenetics/

1. Cosma, M.P. Ordered recruitment: Gene-specific mechanism of transcription activation. Mol. Cell 10, 227-236 (2002).

2. Brown, K.E., Baxter, J., Graf, D., Merkenschlager, M. \& Fisher, A.G. Dynamic repositioning of genes in the nucleus of lymphocytes preparing for cell division. Mol. Cel/3, 207-217 (1999)

3. Gasser, S.M. Positions of potential: Nuclear organization and gene expression. Cell 104, 639-642 (2001).

4. Bird, A.P. DNA methylation patterns and epigenetic memory. Genes Dev. 16, 6-21 (2002).

5. Dillon, N. \& Festenstein, R. Unravelling heterochromatin: competition between posi- tive and negative factors regulates accessibility. Trends Genet. 18, 252-258 (2002).

6. Fisher, A.G. \& Merkenschlager, M. Gene silencing, cell fate and nuclear organisation. Curr. Opin. Genet. Dev. 12, 193-197 (2002).

7. Grewal, S.I. \& Moazed, D. Heterochromatin and epigenetic control of gene expression. Science 301, 798-802 (2003).

8. Lachner, M. \& Jenuwein, T. The many faces of histone lysine methylation. Curr. Opin. Cell Biol. 14, 286-298 (2002).

9. Richards, E.J. \& Elgin, S.C. Epigenetic codes for heterochromatin formation and silencing: rounding up the usual suspects. Cell 108, 489-500 (2002).

10 Fischle, W., Wang, Y. \& Allis, C.D. Histone and chromatin cross-talk. Curr. Opin. Cell Biol. 15, 172-183 (2003).

11. Rusche, L.N., Kirchmaier, A.L. \& Rine, J. The establishment, inheritance, and function of silenced chromatin in Saccharomyces cerevisiae. Annu. Rev. Biochem. 72 481-516 (2003).

12. Wutz, A. \& Jaenisch, R. A shift from reversible to irreversible $X$ inactivation is triggered during ES cell differentiation. Mol. Cel/ 5, 695-705 (2000).

13. Brockdorff, N. X-chromosome inactivation: closing in on proteins that bind Xist RNA. Trends Genet. 18, 352-358 (2002).

14. Mermoud, J.E., Popova, B., Peters, A.H., Jenuwein, T., \& Brockdorff, N. Histone H3 Iysine 9 methylation occurs rapidly at the onset of random $X$ chromosome inactivation. Curr. Biol. 12, 247-251 (2002).

15. Plath, K. et al. Role of histone $\mathrm{H} 3$ lysine 27 methylation in $\mathrm{X}$ inactivation. Science 300, 131-135 (2003).

16. Silva, J. et al. Establishment of histone $\mathrm{H} 3$ methylation on the inactive $\mathrm{X}$ chromosome requires transient recruitment of Eed-Enx1 Polycomb group complexes. Dev. Cel/ 4, 481-495 (2003).

17. Guidos, C.J. Positive selection of $\mathrm{CD}^{+}$and $\mathrm{CD} 8^{+} \mathrm{T}$ cells. Curr. Opin. Immunol. 8 , 225-232 (1996).

18. Turka, L.A. et al. Thymocyte expression of RAG-1 and RAG-2: termination by T cell receptor cross-linking. Science 253, 778-781 (1991).

19. Groves, T. et al. In vitro maturation of clonal $C D 4^{+} C D 8^{+}$cell lines in response to TCR engagement. J. Immunol. 154, 5011-5022 (1995).

20. Groves, T., Parsons, M., Miyamoto, N.G. \& Guidos, C.J. TCR engagement of $\mathrm{CD} 4^{+} \mathrm{CD} 8^{+}$thymocytes in vitro induces early aspects of positive selection, but not apoptosis. J. Immunol. 158, 67-75 (1997).

21. Trinh, L.A. et al. Down-regulation of TDT transcription in $\mathrm{CD}^{+} \mathrm{CD} 8^{+}$thymocytes by Ikaros proteins in direct competition with an Ets activator. Genes Dev. 15, 1817-1832 (2001).

22. Wang, H.B. et al. Purification and functional characterization of a histone H3-lysine 4-specific methyltransferase. Mol. Cell 8, 1207-1217 (2001).

23. Kim, J. et al. Ikaros DNA-binding proteins direct formation of chromatin remodeling complexes in lymphocytes. Immunity 10, 345-355 (1999).

24. Merkenschlager, M. et al. How many thymocytes audition for selection? J. Exp. Med. 186, 1149-1158 (1997). 